\title{
Presente e Futuro da Revista da Sociedade Portuguesa de Dermatologia e Venereologia
}

\section{Present and Future of the Journal of the Portuguese Society of Dermatology and Venereology}

\author{
Helena Donato ${ }^{1,2}$ \\ 'Serviço de Documentação, Centro Hospitalar e Universitário de Coimbra, Coimbra, Portugal \\ ${ }^{2}$ Consultora Técnica da Revista da Sociedade Portuguesa de Dermatologia e Venereologia
}

A qualidade de uma revista científica é frequentemente avaliada pelas bases de dados (BD) onde é indexada e a indexação de uma revista é considerada um reflexo da sua qualidade.

Para ser conhecida como uma fonte relevante de informação científica, e para se destacar de entre muitas outras publicações que estão aglomerando o espaço de publicação, a revista deve aumentar a sua visibilidade, disponibilidade e leitura. Uma das maneiras pelas quais o pode conseguir é sendo indexada pelas bases de dados mais importantes.

O que significa ser uma revista indexada? É uma revista que faz parte de uma base de dados; tem critérios de publicação claros e rigorosos; tem corpo editorial e revisores especializados; tem visibilidade e acessibilidade, mérito e qualidade reconhecidos.

Porque é a indexação essencial?

- A indexação ajuda a revista a atingir seu objectivo principal de estar acessível a um público mais vasto.

- A pesquisa bibliográfica é a primeira actividade que os investigadores realizam como parte do seu estudo e, naturalmente, procuram nas bases de dados mais relevantes. Assim, estar indexada em bases de dados de qualidade reconhecida ajudará a aumentar o número de leitores.

- Estando acessível, por sua vez, melhorará a sua reputação como uma fonte confiável de informação de alta qualidade na sua área e também se tornará mais atractiva para os autores publicarem os seus trabalhos.

- As revistas nacionais que não são indexadas nas principais bases de dados não se tornam atractivas para os autores que precisam de publicar. Estes acabam por enviar os seus trabalhos para as revistas mais prestigiadas, deixando a produção periférica para as revistas nacionais.
Ao pensar sobre indexar a revista, temos de saber que existem várias BD importantes onde a revista da Sociedade Portuguesa de Dermatologia e Venereologia (SPDV) deve estar incluída. Independentemente da base de dados, a revista tem sempre apresentar uma candidatura formal para inclusão, para tal é necessário entender o processo de selecção da base de dados (alguns critérios gerais que todas BD usam para avaliar a adequação da revista para indexação são: qualidade do conteúdo publicado, pontualidade na publicação, qualidade do peer review, fluxo e processo editorial).

Em que BD deve ser a revista indexada?

- Repositório Científico de Acesso Aberto em Portugal (RCAAP)

- SciELO Citation Index (Scientific Electronic Library Online): cobre a literatura biomédica publicada em revistas científicas, incluindo a base de dados SCIE (Science Citation Index-Expanded, Clarivate Analytics)

- DOAJ (Directory of Open Access Journals): é um directório online que indexa e fornece acesso às revistas com peer review de acesso aberto

- Scopus (Elsevier)

- PubMed: inclui a BD bibliográfica Medline e o arquivo de revistas biomédicas em acesso livre, a PMC.

- Emerging Sources Citation Index (ESCI)

- SCIE (Science Citation Index-Expanded), Web of Science, da Clarivate Analytics

- Embase (Elsevier)

- Journal Citation Reports, Clarivate Analytics

A revista da Sociedade Portuguesa de Dermatologia e Venereologia actualmente é indexada no Índex das Revistas
Correspondência: Helena Donato

Serviço de Documentação, Centro Hospitalar e Universitário de Coimbra Praceta Professor Mota Pinto, 3000-075 Coimbra, Portugal

E-mail: helenadonato@chuc.min-saude.pt

DOI: https://dx.doi.org/10.29021/spdv.77.1.1090
Recebido/Received

19 Junho/June 2019

Aceite/Accepted

18 Junho/June 2019 


\section{Editorial}

Médicas Portuguesas (IndexRMP) e no Repositório Científico de Acesso Aberto em Portugal (RCAAP), que para além da literatura científica e académica portuguesa, contém também a produção científica brasileira composta por vários repositórios e revistas agregados no OASIS BR.

A revista submeteu a candidatura à SciELO Citation Index (Scientific Electronic Library Online) e após rigoroso processo de avaliação, foi aceite.

A SciELO, é uma base de dados bibliográfica de referência internacional, integrada na plataforma da Web of Science (WoS) da Clarivate Analytics. Assim, os artigos publicados pela revista SPDV terão contabilizadas as citações que recebem de outros artigos da SciELO. A aceitação na SciELO reflecte qualidade científica dos artigos publicados e destaca o excelente trabalho de editores, autores e revisores.

Web of Science é a designação comum que é dada a um conjunto de bases de dados também conhecidas como Science Citation Indexes, compiladas pela Clarivate Analytics - Thomson Reuters (ex-Institute for Scientific Information).

Para internacionalizar a revista da SPDV vários passos fundamentais têm sido dados. Mas convém realçar que conseguir a indexação em bases de dados de referência internacional é uma missão de todos que exige esforço, tenacidade e capacidade de crítica. Para ganhar um jogo, o primeiro passo é aprender a jogá-lo. Todas as actividades necessitam de uma estratégia e a publicação não é uma excepção à regra. Deve ficar claro que a indexação de uma revista científica é uma aventura com muitos obstáculos difíceis de serem superados e requer uma estratégia contínua, com obrigatoriedade de seguir vários padrões para atingir o nível de excelência internacional.

As diversas BD bibliográficas adoptam critérios de selecção praticamente idênticos, variando apenas o nível de exigência em relação a cada critério: qualidade científica e editorial (incluindo o processo de revisão por pares), corpo editorial, finalidade e cobertura, tipologia do conteúdo, referências actuais e relevantes, credibilidade e qualidade do seu conteúdo, qualidade de produção, regularidade de publicação, diversidade na proveniência dos autores e normalização.'

Várias mudanças têm sido feitas para inovar e internacionalizar a revista da SPDV. Uma série de passos importantes foram dados, e desenvolvemos estas mudanças de acordo com os mais altos padrões éticos e editoriais exigidos para indexação.

Como o idioma pode ser uma limitação para a visibilidade da revista, a revista da SPDV aceita artigos em português mas também em inglês. Publicar em inglês permite captar mais autores assim como alargar o leque de revisores, mas mais do que publicar em inglês é preciso publicar artigos de qualidade, com correcção linguística e gramatical e em acesso aberto.

A reformulação da ficha técnica e do corpo editorial (nacional e internacional) foram as primeiras intervenções efectuadas. Convém realçar que Editor-Chefe (tem de ser sempre alguém de mérito científico reconhecido internacionalmente, pois é o embaixador da revista), Editores-Associados, ConseIho Editorial e Consultores são essenciais e recursos valiosos para a revista. Os seus membros têm de ser figuras proeminentes e respeitadas nas suas áreas, garantindo a qualidade científica do que é publicado na revista e trabalhando para atingir os melhores padrões éticos e de qualidade. ${ }^{2}$ Os revisores fazem um trabalho anónimo e cuidadoso de avaliar os manuscritos, indispensável para que a revista atinja o patamar de qualidade que the permita a indexação.

O segundo passo dado foi garantir que os objectivos e âmbito da revista são claros.

\section{Outros passos foram dados como:}

- Definição das responsabilidades do editor-chefe: foram definidas as responsabilidades do editor-chefe que englobam a implementação da política editorial, a supervisão do processo editorial e as relações da revista com os autores, leitores, indexadores, agências de apoio à investigação, a comunidade científica e o público geral. Em particular, a transparência e o controle de qualidade são aspectos essenciais do processo editorial sob a responsabilidade do editor-chefe.

- Cumprimentos das normas e recomendações de padrões de ética e responsabilidade na comunicação científica estabelecidas pelas instituições internacionais, entre as quais se destacam: Committee on Publication Ethics (COPE), Council of Science Editors (CSE), EQUATOR Network, International Committee of Medical Journal Editors (ICMJE).

- Definição do processo de peer review assim como a opção fast track, passaram a estar claramente descritos nas instruções aos autores, assim como a adesão às recomendações do International Committe of Medical Journals Editors (ICMJE Recommendations), passando a revista a integrar a lista das revistas que cumprem os critérios do ICMJE " journal following the ICMJE recommendations" (http://www.icmie.org/journals-following-the-icmje-recommendations). Também passou a seguir as orientações do EQUATOR Network.

- Qualidade da política editorial e indicação de declaração de liberdade editorial, critérios que também são usados para avaliar a qualidade da revista. ${ }^{3}$

- Promoção da autoria responsável - cumprimento dos critérios de autoria do ICMJE.

- Possui uma política de depósito/copyright no Sherpa/ Romeo.

- Rapidez na publicação através de uma optimização dos tempos editoriais ${ }^{2}$ e o acesso livre aos artigos. ${ }^{4}$

- Aposta na diversidade da distribuição geográfica de autores e colaboradores, pois assim tem maior probabilidade de ter relevância para a comunidade internacional. Internacionalidade: uma revista deve tentar reflectir a diversidade geográfica da área temática pelos autores e conselho editorial.

- Manutenção da pontualidade e a regularidade na publicação: sem atrasos ou interrupções no cronograma de publicação. Não é aceitável que a revista apareça 
cronicamente atrasada, semanas ou meses após a data de capa.

- Obrigatoriedade de publicar estudos negativos, assim como Correcções, Retractações.

- Política de copyright foi definida.

- No que concerne às boas práticas para o fortalecimento da ética na publicação científica, o processo editorial, após o cumprimento dos aspectos formais exigidos, garante que todos os autores reviram e aceitaram a responsabilidade pelo conteúdo e em breve passará a ser exigido que registem a contribuição de cada autor, que será publicado no final do manuscrito. Sendo declarados na publicação a adesão a preceitos éticos e declaração de conflitos de interesse.

- Controlo de práticas antiéticas como a dupla submissão; publicação redundante; autoria inadequada; falta de divulgação de conflitos de interesse e desenvolvimento de directrizes que envolvem o consentimento informado ou privacidade dos doentes.

- Melhoria do trabalho editorial: títulos e resumos descritivos e claros; títulos que reflectem fielmente o conteúdo dos artigos; atribuição das MeSH keywords; actualidade das referências bibliográficas.

- Indicação explícita dos artigos que são sujeitos as peer review externo e inclusão das datas de submissão e de aceitação.

Mas também é necessário promover a revista SPDV:

1. Aumentando a sua visibilidade

2. Consciencializando os autores, líderes de opinião e grupos de trabalho para a qualidade da revista

3. Aumentando o número de submissões nacionais e internacionais

4. Melhorando a qualidade dos artigos publicados

5. Aumentando o respeito por aquilo que a revista publica

6. Aumentando o número de visitas ao website da revista: notificação da publicação de novos números

7. Convidando autores com trabalhos citados a publicar na revista

8. Identificando "hot topics" e convidar a publicar sobre esses temas

9. Aumentando a rapidez na publicação

10. Potenciando o papel dos revisores (peças-chave da qualidade dos trabalhos publicados) criando "normas para revisores"

11. Promovendo a revista na web: nas redes socais genéricas (Facebook; Twitter; LinkedIN) e nas redes sociais académicas (Research Gate; Academia; Mendeley...)

Promovendo a revista é possível captar artigos escritos por autores de outros países e aumentar a diversidade geográfica dos leitores.

A maior e mais prestigiada base de dados bibliográfica continua a ser a Medline, mas apesar de todos os passos que têm sido dados no sentido de demonstrar qualidade do trabalho editorial, incluindo as características que contribuem para a objectividade, credibilidade e qualidade de seu conteúdo, passar a etapa de avaliação de qualificação científica não é fácil e a revista viu a sua candidatura rejeitada. A decisão se uma revista vai ser ou não indexada na Medline é feita pelo director da National Library of Medicine (NLM), baseando-se em parecer emitido por um corpo auxiliar especializado denominado Literature Selection Technical Review Committee (LSTRC). O LSTRC é composto por quinze membros ligados aos National Institutes of Health, e reúne-se três vezes ao ano. ${ }^{5}$ Aproximadamente 160 revistas são examinadas em cada reunião. Nas reuniões são analisados os quatro últimos números publicados de cada título. Em média, de $12 \%$ a $15 \%$ das revistas avaliadas em cada reunião são recomendadas para inclusão no MEDLINE. Uma revista como a revista SPDV que publica 4 números por ano, nesses 4 números terá de ter publicado no mínimo 12 originais.

Os pontos mais valorizados pelo Literature Selection Technical Review Committee da Medline são ${ }^{5}$ :

1. Finalidade e Cobertura

- Política editorial convincente

- Diversidade na proveniência do conselho editoral

- Diversidade na proveniência dos autores

2. Qualidade de conteúdo - mérito científico do conteúdo é a principal consideração na selecção para indexação

- Validade

- Relevância

- Originalidade e actualidade

- Fiabilidade e validade científica

- Apresentação (boa redacção - clara, concisa, precisa; boa organização - coerência lógica)

- Contribuição para a área

3.Tipologia do conteúdo (prioridade: próxima da ordem em que a tipologia está listada) e Referências actuais e relevantes

- Original Research

- Original clinical observations accompanied by analysis and discussion

- Analysis of philosophical, ethical, social aspects of the health professions or biomedical sciences

- Reviews

- Statistical compilations

- Descriptions of evaluation of methods or procedures

- Case reports with discussions

4. Seguir as orientações internacionais:

- International Committee of Medical Journal Editors (ICJME)

- Committee on Publication Ethics (COPE)

- EQUATOR Network

- Council of Science Editors (CSE) "Editorial Policy Statements

5. Qualidade do trabalho editorial

- Analisam os protagonistas da revista: autores e responsáveis científicos (avaliam se equipa editorial publica artigos em revistas de mérito cientifico, se participam em outras revistas científicas)

- Credibilidade e qualidade do seu conteúdo 


\section{Editorial}

- Títulos e Resumos descritivos e em inglês

- MeSH keywords

- Informações sobre os métodos de selecção de artigos, especialmente sobre o processo explícito de revisão por pares

- Declarações de adesão a preceitos éticos

- Conflitos de Interesse

- Correcção atempada - erratas e retractações

- Declaração de Contribuição - exigir que cada manuscrito submetido tenha "Contributorship Statement"

- Divulgação de conflitos de interesse

- Controle de práticas antiéticas

- Indicação explicita dos artigos sujeitos as peer review externo

- Patrocínio comercial não deve levantar questões sobre a objectividade do material publicado

6. Qualidade de produção - Qualidade do layout, impressão, figuras/tabelas, impressão em papel acid-free

\section{Audiência e atracção}

- Audiência - indicadores sobre atracção, visibilidade da revista

- Indicadores:

- Distribuição

- Procedência dos autores

- Indexação em bases de dados

- Presença na Internet

- Capitalizar as oportunidades oferecidas pela publicação online - promovendo a visibilidade na web social e colaborativa

Mas para estar presente na PubMed, existe uma alternativa à indexação na Medline. A outra via é a indexação na PubMed Central (PMC)

PMC é um repositório digital de acesso aberto desenvolvido e mantido pelo National Center for Biotechnology Information (NCBI) / Nacional Library of Medicine (NLM), que reúne artigos científicos da área de biomedicina e ciências da vida, publicados em inglês. ${ }^{6}$

Para participar no PMC a revista terá de ser editada só em inglês e em open access. Ou seja terá de ser criada uma nova revista, com um novo ISSN, e só após mais de 2 anos de publicação e um mínimo de 25 artigos revistos por pares publicados poderá ser feita a candidatura.

A candidatura inicia-se com o Submit Application em que terão de ser fornecidos os seguintes $\operatorname{dados}^{6}$ :

- Título completo da revista e ISSN

- Quando a revista começou a ser publicada e periodicidade

- URL para o site da revista

- Links directos para o conselho editorial, políticas editoriais, processo de revisão por pares e políticas sobre Conflito de Interesses, Direitos Humanos e Animais e Consentimento Livre e Esclarecido

- Nome da Editora (publisher) e informações sobre políticas de publicação
Após uma revisão completa das informações, políticas e conteúdo da revista, a PMC informa o editor se a revista cumpre ou não aos padrões de qualidade científicos e editoriais do NLM para inclusão na PMC.

Se a revista passar a avaliação scientific quality standard é solicitado o envio de um conjunto representativo de artigos de arquivo como amostra, que são avaliados para garantir que os dados da revista atendem aos padrões técnicos de qualidade da PMC.

PMC exige:

- Separate XML data file for the full text of each article

- Original high-resolution digital image files for all figures in each article

- $A P D F$, if one exists, in addition to the XML version (but not as the only form)

- Supplementary data files (e.g., spreadsheets or video files) available with the article

Outro excelente recurso é o ESCl (Emerging Sources Citation Index). É uma base de dados que compõe a colecção do Web of Science e indexa revistas emergentes que ainda não receberam o factor de impacto do Journal Citation Reports (JCR).

Estas revistas são avaliadas e seleccionadas, atendendo a padrões de qualidade editorial, oportunidade e impacto. Após um período poderá ser indexada nas colecções: Science Citation Index Expanded (SCI-EXPANDED)

$\bigcirc \mathrm{ESCl}$ propõe ampliar conteúdos regionais e campos emergentes gerando visibilidade para revistas e autores, permitindo que as revistas indexadas sejam pesquisáveis, reconhecidas e citáveis. A inclusão no $\mathrm{ESCl}$ proporciona uma maior descoberta que leva a citações mensuráveis e mais transparência no processo de selecção.

grande objectivo desta indexação é promover a presença da revista da SPDV em um dos índices bibliográficos e bibliométricos de referência internacional para ampliar a visibilidade e credibilidade da revista. Também se pretende com esta indexação contabilização das citações que os artigos da revista da SPDV recebem em outros artigos de revistas da WoS e desta forma vir a ser considerada para inclusão no Journal Citation Reports.

A Scopus é uma base de dados multidisciplinar de grande prestígio, com grande cobertura para a área médica. É uma base de dados editada pela holandesa Elsevier e como tal favorece a indexação de revistas europeias. Tornar-se parte do Scopus facilita a visibilidade dos autores e seus artigos, o que torna as revistas que a integram mais atraentes para publicação. A revista da SPDV pode avançar para a candidatura para alcançar essa inclusão. $O$ processo de avaliação e comunicação às partes interessadas leva um ano e, durante esse tempo, avaliarão vários aspectos como se a revista está em conformidade com o controle do plágio, se age com base em políticas éticas, se realiza revisão por pares dos artigos, a internacionalização ou o prestígio dos autores e do conselho editorial. 
Para concretização do grande salto qualitativo, a indexação, tem de existir um aumento no número e qualidade dos artigos submetidos, tem de haver uma maior contribuição individual do corpo editorial, a qualidade do conteúdo é primordial assim como demonstrar a capacidade de manter esse status de qualidade. Mais importante que publicar um bom número é manter constante a qualidade científica, editorial e de produção (gráfica e informativa), assim como a pontualidade. Pois será a constância e pontualidade que garantirão a credibilidade e respeito a nível internacional. ${ }^{7}$

Como conclusão é possível afirmar que o que realmente faz uma revista de alta qualidade é primeiro e acima de tudo a competência e dedicação dos seus editores e revisores, bem como a excelência do seu processo editorial. $O$ trabaIho de conjunto entre a equipa editorial e revisores altamente dedicados e motivados atrairá mais autores e melhores trabalhos, que permitirão atingir o nível de excelência que levará à indexação em bases de dados de grande relevância. Depende de todos, editores, revisores e autores alcançar a tão sonhada excelência da indexação.

Quantas mais forem as bases de dados relevantes onde a revista está visível, maior é a oportunidade de quem nela publica construir uma reputação sólida na área.

Acreditamos que estamos no caminho certo. Mas, como sempre, precisamos de todos os dermatologistas nacionais se comprometam nesta cruzada se queremos colocar a RSPDV no futuro!

Conflitos de interesse: Os autores declaram não possuir conflitos de interesse.

Suporte financeiro: $O$ presente trabalho não foi suportado por nenhum subsidio o bolsa ou bolsa.

Conflicts of interest: The authors have no conflicts of interest to declare.

Financial support: This work has not received any contribution grant or scholarship.

Proveniência e revisão por pares: Não comissionado; revisão externa por pares.

Provenance and peer review: Not commissioned; externally peer reviewed.

\section{REFERÊNCIAS}

1. Donato H. Publicações médicas nacionais: passado, presente e futuro. Rev Soc Port Med Interna. 2015; 22:5-7.

2. Sanclemente G, Pardo H, Sánchez S, Bonfill X. Analysis of the quality of clinical trials published in Spanish-language dermatology journals between 1997 and 2012. Actas Dermosifiliogr. 2016;107:44-54.

3. Kanter SL. Understanding the Journal's Impact. Acad Med. 2009;84:1 169-70.

4. Eysenbach $G$. The open access advantage. J Med Internet Res. 2006;8:e8.

5. National Center for Biotechnology Information, National Library of Medicine. Journal Selection for MEDLINE ${ }^{\circledR}$ Indexing at NLM [consultado 14 Junho] Disponível em: https://www.nlm.nih.gov/Istrc/i_sel_faq.html

6. National Center for Biotechnology Information, National Library of Medicine. How to Include a Journal in PMC. [consultado 14 Junho] Disponível em: https://www.ncbi. nlm.nih.gov/pmc/pub/addjournal

7. Donato H. Caminhos para a indexação. Rev Soc Port Med Interna.2016;23:3-4. 